\title{
Dual anti-cancer effects of weekly intraperitoneal docetaxel in treatment of advanced gastric cancer patients with peritoneal carcinomatosis: A feasibility and pharmacokinetic study
}

\author{
SACHIO FUSHIDA, JUN KINOSHITA, YASUMICHI YAGI, \\ HIROSHI FUNAKI, SHINICHI KINAMI, ITASU NINOMIYA, TAKASHI FUJIMURA, \\ GENICHI NISHIMURA, MASATO KAYAHARA and TETSUO OHTA \\ Department of Gastroenterologic Surgery, Kanazawa University Hospital, Kanazawa, Japan
}

Received December 21, 2007; Accepted February 8, 2008

\begin{abstract}
This study evaluated the feasibility and pharmacology of intraperitoneal docetaxel (IP docetaxel) when administered weekly for 3 consecutive weeks, followed by 1 week without treatment. A total of 24 patients with peritoneal carcinomatosis of gastric cancer (10 preoperative, 7 postoperative and 7 recurrent) were enrolled in this study. Docetaxel was dissolved in an isotonic saline to a final 1 liter solution and was administered in a $1 \mathrm{~h}$ dosage of 25, 35, 45 and $60 \mathrm{mg} / \mathrm{m}^{2}$ to determine the maximum tolerated dose (MTD). To measure the docetaxel concentration, blood and peritoneal fluid samples were collected $0.5,1,2,3,6$ and $24 \mathrm{~h}$ after administering the drug to 15 patients. A total of 109 chemotherapy cycles were administered, with a median of four cycles per patient (range 2-9). The MTD of the weekly IP docetaxel was defined at $60 \mathrm{mg} / \mathrm{m}^{2}$. At a docetaxel dosage of $60 \mathrm{mg} / \mathrm{m}^{2}$ per week, the dose-limiting events of grade 3 abdominal pain and grade 3 diarrhea, which may be associated with local toxicity, occurred. Peak concentrations of peritoneal fluid ranged from 24.5 to $68.7 \mu \mathrm{g} / \mathrm{ml}$. The mean ratio of the area under concentration (AUC) in the peritoneal fluid to the plasma concentration was 515. Furthermore, the mean of plasma AUC by IP docetaxel was $5.63 \mu \mathrm{g} \cdot \mathrm{h} / \mathrm{ml}$ versus that of IV docetaxel at a dose of $60 \mathrm{mg} / \mathrm{m}^{2}$. The response rate of the preoperative IP docetaxel was $80 \%$ (4 CR, 4 PR, $1 \mathrm{NC}$ and 1 PD), which was judged with laparoscopy and peritoneal lavage cytology. Gastrectomy, with D2 lymph node dissection, was performed on all of the patients evaluated as CR. The weekly IP docetaxel demonstrated a low toxicity and high efficacy for peritoneal carcinomatosis with dual anti-cancer effects via the peritoneal surface and capillary blood supply due to its unique pharmacokinetic property.
\end{abstract}

Correspondence to: Dr Sachio Fushida, Department of Gastroenterologic Surgery, Kanazawa University Hospital, Takaramachi 13-1, Kanazawa 920-8641, Japan

E-mail: fushida@surg2.m.kanazawa-u.ac.jp

Key words: intraperitoneal docetaxel, peritoneal carcinomatosis, gastric cancer

\section{Introduction}

One of the most characteristic features and frequent causes of death from gastric cancer is peritoneal dissemination. In a recent multicentric prospective study (1), the median survival time was 3.1 months for gastric cancer patients. For two decades now, the treatment of peritoneal dissemination has consisted of systemic chemotherapy with sequential methotrexate (MTX) and 5-FU, or intraperitoneal chemotherapy (IPC) with mitomycin C (MMC), cisplatin (CDDP), OK-432 and other agents. Sequential MTX and 5-FU have been widely used as systemic chemotherapy because of their high efficacy against poorly-differentiated adenocarcinoma, persisting high concentrations in ascites and a tendency to have low-grade toxicity $(2,3)$. However, gastric cancer is only moderately sensitive to chemotherapy and peritoneal metastasis is known to be relatively resistant to systemic chemotherapy due to the poor blood supply and oxygenation of cancer cells in the peritoneum. Therefore, to enhance the efficacy of anti-cancer drugs, IPC has generally been accepted as a regional intensive chemotherapy for the prevention and treatment of peritoneal dissemination.

IPC with MMC or carbon-adsorbed MMC has been reported to improve the survival of gastric cancer patients by preventing peritoneal recurrence $(4,5)$, but the efficacy of these therapies in patients with peritoneal dissemination has yet to be established. Intraperitoneal CDDP treatment has been performed safely and effectively in ovarian cancer patients by many investigators $(6,7)$.

The usefulness of intraperitoneal CDDP has to be confirmed by controlled clinical studies. However, a multicenter randomized trial, JCOG 9701 (Japan Clinical Oncology Group), which aimed to compare adjuvant intraperitoneal CDDP with no adjuvant treatment, was stopped because of the difficulty of randomizing patients into the no treatment group.

Although three large randomized phase III trials comparing IP vs. IV cisplatin-based chemotherapy have shown a survival benefit of IPC, this approach has yet to be accepted as a standard treatment for gynecological tumors (8-10).

The efficacy of IPC is mainly affected by the extent of the peritoneal tumor and ascites. Most of the positive results were obtained for patients without peritoneal dissemination in an 
adjuvant setting or for patients with microscopic residual tumors, even if hyperthermia was added in anticipation of its synergy effect (11-17).

Taxanes such as docetaxel and paclitaxel bind to tubulin, leading to microtubule stabilization, mitotic arrest and, subsequently, cell death $(18,19)$. The activity of taxanes may depend on the property of killing tumor cells in the absence of the wild-type p53 function (20), unlike other drugs requiring wild-type p53, and taxanes may therefore be effective against gastric cancer cells, which frequently have p53 mutations $(21,22)$. Furthermore, these compounds have a high sensitivity against poorly-differentiated adenocarcinoma, which is a common type of peritoneal tumor, and some of these compounds, when administered intravenously, are transported into the peritoneal cavity $(23,24)$. These findings suggest that taxanes are also candidates for first-line drugs for peritoneal carcinomatosis.

Several studies of intraperitoneal paclitaxel showed a minimal toxicity and pharmacokinetic advantage. However, few reports about the feasibility, pharmacokinetics and efficacy of IP docetaxel exist. In this study, we examined the disposition kinetics of docetaxel and paclitaxel after intraperitoneal administration in patients with peritoneal carcinomatosis of gastric cancer.

\section{Patients and methods}

Patients. The patients with cytologically or histologically confirmed peritoneal carcinomatosis were eligible for this study. Other eligibility criteria were: age 18-75 years, Eastern Cooperative Oncology Group (ECOG) performance status 0-2, no history of previous chemo- or radiotherapy, adequate bone marrow function $\left(<3000 / \mathrm{mm}^{3}\right.$ white cell count or $<1500 / \mathrm{mm}^{3}$ neutrophil count, platelet count $>80,000 / \mathrm{mm}^{3}$ and hemoglobin $>8.0 \mathrm{~g} / \mathrm{dl}$ ), adequate liver function (bilirubin level $<1.5 \mathrm{mg} / \mathrm{dl}$, and aspartate and alanine aminotransferase within twice the upper limit of the normal). All of the patients gave written informed consent, conforming to institutional guidelines.

Treatment plan. The docetaxel (Taxotere ${ }^{\circledR}$, Sanofi-Aventis Ltd.) dose was dissolved at room temperature in an isotonic saline to a final 1 liter solution and was instilled into a peritoneal cavity through the catheter for $1 \mathrm{~h}$. Prior to therapy, patients received dexamethasone and the $5-\mathrm{HT}_{3}$ antagonist intravenously $30 \mathrm{~min}$ before the taxane to prevent hypersensitivity reactions. After instillation, patients were asked to lie on a bed and change positions at 30-min intervals for $24 \mathrm{~h}$ to ensure adequate intraperitoneal drug distribution.

The standard dosing regimen for docetaxel is $75-100 \mathrm{mg} / \mathrm{m}^{2}$ administered every 3 weeks. In this study, patients received weekly IP docetaxel for 3 consecutive weeks, followed by 1 week without treatment because several clinical studies showed that a weekly administration of docetaxel achieves the equivalent efficacy of a 3 -week schedule $(25,26)$. The initial starting dose of docetaxel was $25 \mathrm{mg} / \mathrm{m}^{2}$ (dose level 1) and step-wise dose increases up to $60 \mathrm{mg} / \mathrm{m}^{2}$ were planned for successive patient cohorts.

At least three assessable patients were treated at each dose level and an assessment of DLTs was conducted only in the first treatment cycle. If one or more patients at a dose level experienced DLT, then three additional patients were treated at that dose level. The MTD was defined as the dose level that produced DLT in $\geq 3$ patients or the initial 3 patients. The recommended dose was to be the dose immediately below the MTD. Toxicity was measured by the common toxicity criteria of the National Cancer Institute, version 2.0.

Pharmacokinetic analysis. To measure the docetaxel and paclitaxel concentration, blood and peritoneal fluid samples were collected whenever possible before administration, 0.5 , 1, 2, 3, 6 and $24 \mathrm{~h}$ after drug instillation ended. Samples were collected in tubes containing EDTA, centrifuged and the supernatant was stored at $-20^{\circ} \mathrm{C}$ until assay. The assays for docetaxel and paclitaxel were performed according to Loss et al (27). All samples were analyzed on an HPLC system and the absorbance was detected at a wavelength of $230 \mathrm{~nm}$. The limit of quantification was $\sim 0.05 \mathrm{mg} / \mathrm{ml}$.

Pharmacokinetic parameters were calculated using the one-compartment model according to Kuzuya et al (28). The area under the drug concentration-time curve from $0-24 \mathrm{~h}$ in the peritoneal fluid (AUCper, 0-24 h) or in plasma (AUCpl, 0-24 h) was estimated by using the trapezoidal method.

Response criteria. Peritoneal dissemination is considered to be a non-evaluated lesion because it was difficult to detect peritoneal dissemination by conventional radiological examinations. In this study, defined new response criteria for treatment against peritoneal dissemination were followed (Table I): $\mathrm{CR}$, no visual peritoneal tumor confirmed by laparoscopy and cytology negative; PR, at least a 50\% decrease of peritoneal tumor confirmed by laparoscopy or cytology negative; SD, no unequivocal progression of peritoneal dissemination and $\mathrm{PD}$, unequivocal progression of peritoneal dissemination.

\section{Results}

Patient characteristics. Between June 2000 and October 2004, 24 patients with peritoneal dissemination of gastric cancer were entered in this study. The patient characteristics are listed in Table II. Peritoneal carcinomatosis, was classified by the Japanese Research Society for Gastric Cancer (29) as: $\mathrm{P} 1$, cancerous implants to the region directly adjacent to the stomach peritoneum (above the transverse colon) including the great omentum; P2, several scattered metastases to the distant peritoneum and ovarian metastasis alone and P3, numerous metastases to the distant peritoneum. P1 was observed in 3 patients, $\mathrm{P} 2$ in 8 patients and $\mathrm{P} 3$ in 13 patients. Seven of the postoperative patients were diagnosed with peritoneal dissemination at laparotomy and underwent cytoreductive surgery, the remainder (10 preoperative and 7 recurrent) had to undergo laparoscopy for diagnosis. The total course of IP docetaxel was 109 and the median course was 4 (range 2-9).

Toxicity. To evaluate the local toxicity of the treatment, an abdominal pain score was used, originally applied in the phase I study of intraperitoneal paclitaxel (30) (Table III). No local toxicity was evident at dose level $1\left(25 \mathrm{mg} / \mathrm{m}^{2}\right)$ but a mild one was detected at dose level $2\left(35 \mathrm{mg} / \mathrm{m}^{2}\right)$. At a dose level of $4\left(60 \mathrm{mg} / \mathrm{m}^{2}\right), 2$ out of 3 patients had severe abdominal pain 
Table I. New response criteria for treatment against peritoneal carcinomatosis.

Complete response (CR)

Partial response $(\mathrm{PR})$

Stable disease $(\mathrm{SD})$

Progressive disease (PD)
No visual peritoneal tumor (by laparoscopy or laparotomy)

Cytology (lavage cytology) negative

$<50 \%$ of residual peritoneal tumor

Cytology (lavage cytology) negative

No unequivocal progression of peritoneal carcinomatosis

Unequivocal progression of peritoneal carcinomatosis
Table II. Patient characteristics.

Gender (F/M)

Performance status $(0 / 1 / 2)$

$15 / 9$

Timing of chemotherapy

(preoperative / postoperative / recurrent)

$12 / 10 / 2$

Peritoneal carcinomatosis (P1/P2/P3)

$10 / 7 / 7$

Ascites $\left(0 / 1^{+} / 2^{+} / 3^{+}\right)$

Table III. Abdominal pain score.

\begin{tabular}{ll}
\hline Grade & \multicolumn{1}{c}{ Performance } \\
\hline 0 & No pain \\
1 & Mild pain \\
& Narcotic analgesia not required \\
& Minimal interference with daily activities \\
& lasts for $<72 \mathrm{~h}$ \\
& Moderate pain \\
& Narcotic analgesia not required \\
& Moderate interference with daily activities \\
& lasts for $>72 \mathrm{~h}$ \\
& Seve pain \\
& Narcotic analgesia required \\
& Confines patient to bed \\
& Severe interference with daily activities
\end{tabular}

and the other one had grade 3 diarrhea (Table IV). Diarrhea was relatively frequent, with grade 2 diarrhea being observed in 4 patients and grade 3 in 1 patient which may have induced intestinal edema as local toxicity. Nausea and/or vomiting were mild and easily controlled. Bone marrow suppression was also mild.

From these results, the MTD and RD were determined to be level 4 and 3 , respectively.

Pharmacokinetic results. Abdominal fluid and plasma samples were obtained from 15 patients at a dose level of $3\left(45 \mathrm{mg} / \mathrm{m}^{2}\right)$, which was determined to be the recommended dose. The pharmacokinetic analyses are summarized in Table V. The peak concentrations of the peritoneal fluid ranged from 24.5
Table IV. Local and systemic toxicity associated with IP docetaxel.

\begin{tabular}{|c|c|c|c|c|}
\hline \multirow{3}{*}{$\begin{array}{l}\text { Toxicity grade } \\
\text { No. of evaluated patients }\end{array}$} & \multicolumn{4}{|c|}{ Dose level $\left(\mathrm{mg} / \mathrm{m}^{2}\right)$} \\
\hline & 25 & 35 & 45 & 60 \\
\hline & 3 & 3 & 15 & 3 \\
\hline \multicolumn{5}{|l|}{ Abdominal pain } \\
\hline 0 & 3 & 2 & 11 & 0 \\
\hline 1 & 0 & 1 & 2 & 0 \\
\hline 2 & 0 & 0 & 2 & 1 \\
\hline 3 & 0 & 0 & 0 & 2 \\
\hline \multicolumn{5}{|l|}{ Diarrhea } \\
\hline 0 & 3 & 2 & 11 & 0 \\
\hline 1 & 0 & 1 & 1 & 1 \\
\hline 2 & 0 & 0 & 3 & 1 \\
\hline 3 & 0 & 0 & 0 & 1 \\
\hline \multicolumn{5}{|l|}{ Leucocytopenia } \\
\hline 0 & 3 & 2 & 11 & 0 \\
\hline 1 & 0 & 1 & 3 & 2 \\
\hline 2 & 0 & 0 & 1 & 1 \\
\hline 3 & 0 & 0 & 0 & 0 \\
\hline \multicolumn{5}{|l|}{ Alopecia } \\
\hline 0 & 3 & 3 & 12 & 1 \\
\hline 1 & 0 & 0 & 2 & 1 \\
\hline 2 & 0 & 0 & 1 & 1 \\
\hline
\end{tabular}

to $68.7 \mu \mathrm{g} / \mathrm{ml}$. The mean ratio of AUC in the peritoneal fluid to plasma concentration was 515 (Table V). The mean value of the peak plasma concentration was extremely low versus that of the abdominal fluid but the mean value of plasma AUC was $5.63 \mu \mathrm{g} \cdot \mathrm{h} / \mathrm{ml}$ which was equivalent to that of intravenous docetaxel (31) (Table VI).

Efficacy. The response rate of the preoperative intraperitoneal docetaxel was $80 \%$ (4 CR, 4 PR, 1 NC and 1 PD), which was judged with laparoscopy and peritoneal lavage cytology. Gastrectomy, with D2 lymph node dissection, was performed on all the patients evaluated as CR. Of the patients that underwent gastrectomy against the $\mathrm{T} 3$ tumor for postoperative intraperitoneal docetaxel, 6 out of 7 had no peritoneal recurrence after the gastrectomy. However, a lower response 


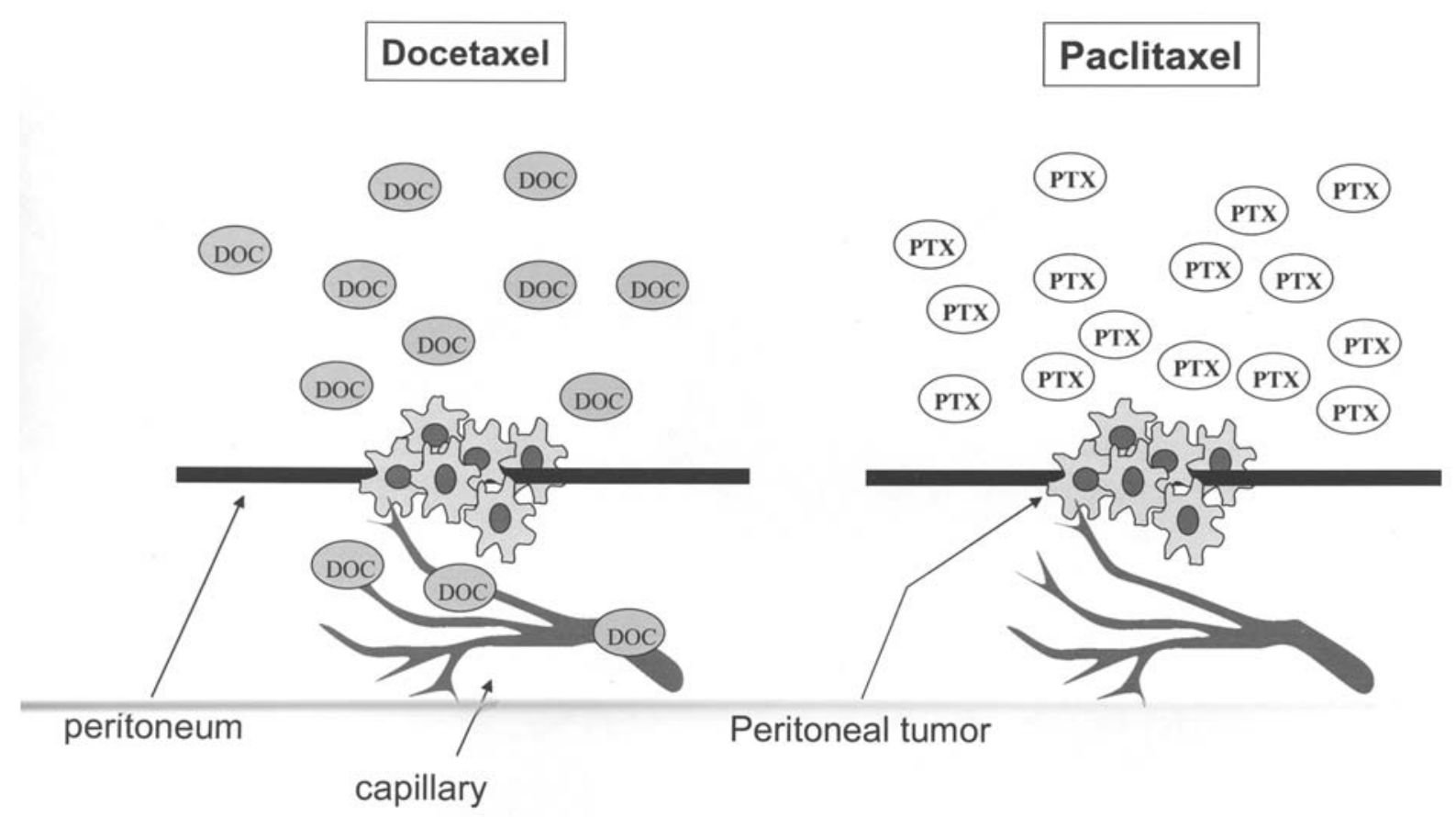

Figure 1. The kinetic disposition between docetaxel and paclitaxel after intraperitoneal administration. Docetaxel may affect the peritoneal tumor via the peritoneal surface and capillary blood supply due to its unique pharmacokinetic property.

Table V. Peritoneal pharmacokinetics.

Mean peak peritoneal concentration

Mean peritoneal AUC

MeanAUC peritoneal $\mathrm{AUC}_{\text {plasma }}$
$40.1 \mu \mathrm{g} / \mathrm{ml}(24.5-68.7)$

$473.6 \mu \mathrm{g} \cdot \mathrm{h} / \mathrm{ml}(197.3-1278.2)$

515 (22-1773)
Table VI. Plasma pharmacokinetics.

$\begin{array}{cc}\text { IV docetaxel } & \text { IP docetaxel } \\ \left(60 \mathrm{mg} / \mathrm{m}^{2}\right) & \left(45 \mathrm{mg} / \mathrm{m}^{2}\right)\end{array}$

Mean peak plasma

Concentration $(\mu \mathrm{g} / \mathrm{ml})$

Mean plasma AUC

$(\mu \mathrm{g} \cdot \mathrm{h} / \mathrm{ml})$

2.44

5.63

rate $(57 \%)$ was found in the patients with peritoneal recurrence than in the preoperative ones.

\section{Discussion}

The pharmacokinetic properties of many anti-cancer agents have been examined, following intraperitoneal administration in phase I studies. The ideal agents for intraperitoneal delivery have a high ratio of either a peak peritoneal drug concentration or area under the peritoneal concentration versus the time curve (AUC) relative to systemic concentration, i.e., they have a low peritoneal but a high plasma clearance. Such a pharmacokinetic advantage for peritoneal cavity exposure is favored by high molecular weight, water solubility, high solution volume and easy ionization. The pharmacokinetic advantage has been reported to range from 20 -fold for cisplatin (7) and carboplatin (32), to as high as 1000-fold for paclitaxel (30).

Since mitomycin C, commonly used intraperitoneally, is rapidly absorbed through the capillary walls in the subperitoneum due to its low solubility in water, mitomycin $\mathrm{C}$ has only a 20-30-fold pharmacokinetic advantage and disappears from the blood within $3 \mathrm{~h}$ (33).

Cisplatin, whose molecular weight is relatively low, is also rapidly absorbed by the subperitoneal capillaries and transported into the systemic circulation. Pharmacokinetic studies confirmed that the plasma AUC is similar after the intraperitoneal or intravenous administration of cisplatin. The adverse effects after the intraperitoneal administration of cisplatin are similar to those after intravenous administration, and include effects such as emesis, nephrotoxicity and neurotoxicity, with minimal local adverse events. Considered together, these findings indicate that intraperitoneally administered cisplatin may exert anti-cancer effects regionally and systemically (34). However, it has been shown that cisplatin is useful for patients with microscopic but not 
macroscopic residual tumors. Therefore, it is necessary to search for another highly effective agent for intraperitoneal administration in gastric cancer.

Paclitaxel is retained in the peritoneal cavity at cytotoxic concentrations for at least 7 days, implying that very limited amounts of paclitaxel enter the systemic compartment after intraperitoneal administration (35). This is reasonable, considering that the dose-limiting factor for paclitaxel is abdominal pain from direct peritoneal irritation. These findings suggest that the cytotoxic activity of intraperitoneal paclitaxel is exerted by direct penetration into the regional tumor alone.

In contrast, docetaxel has a pharmacokinetic advantage of two logs associated with its intraperitoneal delivery, and the systemic AUC after intraperitoneal administration is two times greater than after standard intravenous administration (36). These data indicate that docetaxel occupies a position between cisplatin and paclitaxel from the pharmacokinetic viewpoint (Fig. 1). Paclitaxel and docetaxel have similar chemical and physiological characteristics, and the pharmacokinetic difference between them seems to be attributable to the differential absorption in solubility (36). The injection preparation of paclitaxel, Taxol, contains a high concentration of Cremophor EL as the surfactant vehicle, which suppresses the permeation of the anti-cancer drug into tissues and cells, which may explain why intraperitoneally administered Taxol shows a lower systemic transportation than that of Taxotere, which contains a low concentration of Polysorbate- 80 as the surfactant (37).

The present study suggested that it is feasible to use docetaxel at a dose level of $45 \mathrm{mg} / \mathrm{m}^{2}$. The response rate of this study was $70 \%$ in 17 valuable cases and 4 cases were evaluated as CR, while Morgan et al (37) reported that there were no objective responses to $100 \mathrm{mg} / \mathrm{m}^{2}$ of IP docetaxel every 3 weeks. This weekly administration appeared to have improved results because of this high dose intensity compared to the usual 3-week administration. Furthermore, weekly IP docetaxel may also have shown the anti-cancer effect as being weekly intravenously (IV) docetaxel, because the systemic AUC of the IP docetaxel was comparable to that of the IV docetaxel whose pharmacokinetic properties are well described (31).

This study showed that weekly IP docetaxel is the best treatment for peritoneal carcinomatosis with dual anti-cancer effects via the peritoneal surface and capillary blood supply.

\section{References}

1. Sadeghi B, Arvieux C, Glehen O, et al: Peritoneal carcinomatosis from non-gynecologic malignancies: results of the Evocape 1 multicentric prospective study. Cancer 88: 360-365, 2000.

2. Konishi T, Noie T, Yoshida J, Mafune K and Makuuchi M: Treatment of peritoneal dissemination of gastric cancer with sequential methotrexate and 5-fluorouracil. Gastric Cancer 2: 52-56, 1999.

3. Tahara M, Ohtsu A, Boku N, et al: Sequential methotrexate and 5-fluorouracil therapy for gastric cancer patients with peritoneal dissemination: a retrospective study. Gastric Cancer 4: 212-218, 2001.

4. Hiratsuka M, Ogawa A, Ohigashi H, et al: Dosage and its safety on intraperitoneal-high dose mitomycin $\mathrm{C}$ administration as postoperative adjuvant therapy for gastric cancer. J Jpn Soc Cancer Ther 23: 1574-1579, 1998.

5. Hagiwara A, Takahashi T, Kojima O, et al: Prophylaxis with carbon-adsorbed mitomycin against peritoneal recurrence of gastric cancer. Lancet 339: 629-631, 1992.
6. Howel SB: Intraperitoneal chemotherapy for ovarian carcinoma. J Clin Oncol 6: 1673-1675, 1988.

7. Casper ES, Kelsen DP, Alcock NW and Lewis JL Jr: Ip cisplatin in patients with malignant ascites: pharmacokinetic evaluation and comparison with the IV route. Cancer Treat Rep 67: 235-238, 1983

8. Alberts DS, Liu PY, Hannigan EV, et al: Intraperitoneal cisplatin plus intravenous cyclophosphamide versus intravenous cisplatin plus intravenous cyclophosphamide for stage III ovarian cancer. N Engl J Med 335: 1950-1955, 1996.

9. Markman M, Bundy BN, Alberts DS, et al: Phase III trial of standard-dose intravenous cisplatin plus paclitaxel versus moderately high-dose carboplatin followed by intravenous paclitaxel and intraperitoneal cisplatin in small-volume stage III ovarian carcinoma: an intergroup study of the gynecologic oncology group, Southwestern oncology group, and Eastern cooperative oncology group. J Clin Oncology 19: 1001-1007, 2001.

10. Armstrong DK, Bundy BN, Wenzel L, et al: Intraperitoneal cisplatin and paclitaxel in ovarian cancer. N Engl J Med 354: 34-43, 2006.

11. Spratt JS, Adock RA, Muskovin M, Sherrill W and McKeown J: Clinical delivery system for intaperitoneal hyperthermic chemotherapy. Cancer Res 40: 256-260, 1980.

12. Teicher BA, Kowai CD, Kennedy KA and Sartorelli AC: Enhancement by hyperthermia of the in vitro cytotoxicity of mitomycin $C$ toward hypoxic tumor cells. Cancer Res 41: 1096-1099, 1981.

13. Hamazoe R, Maeta M and Kaibara N: Intraperitoneal thermochemotherapy for prevention of peritoneal recurrence of gastric cancer. Final results of a randomized controlled study. Cancer 73: 2048-2052, 1994.

14. Fujimura T, Yonemura Y, Muraoka K, et al: Continuous hyperthermic peritoneal perfusion for the prevention of peritoneal recurrence of gastric cancer. World J Surg 18: 150-155, 1994.

15. Takahashi T, Hagiwara A, Shimotsuma M, Sawai K and Yamaguchi T: Prophylaxis and treatment of peritoneal carcinomatosis: intraperitoneal chemotherapy with mitomycin $\mathrm{C}$ bound to activated carbon particles. World J Surg 19: 565-569, 1995.

16. Yu W, Whang I, Chung HY, Averbach A and Sugarbaker PH: Indications for early postoperative intraperitoneal chemotherapy of advanced gastric cancer: results of a prospective randomized trial. World J Surg 25: 985-990, 2001.

17. Yonemura Y, Fujimura T, Nishimura G, et al: Effects of intraoperative chemohyperthermia in patients with gastric cancer with peritoneal dissemination. Surgery 119: 437-444, 1996.

18. Rowinsky EK, Cazenave LA and Donehower RC: Taxol: a novel investigational antimicrotubule agent. J Natl Cancer Inst 82: 1247-1259, 1990.

19. Ringel I and Horwits B: Studies with RP56976 (Taxotere): a semisynthetic analogue of taxol. J Natl Cancer Inst 83: 288-291, 1991.

20. Wahl AF, Donaldson KL and Fairchild C: Loss of normal p53 function confers sensitization to taxol by increasing G2/M arrest and apoptosis. Nat Med 2: 72-79, 1996.

21. Fushida S, Urano T, Tsuruta H, Yonemura Y, Miyazaki I and Shiku H: Frequent p53 mutations on exons 5 and 8 in human primary gastric cancer. Int J Oncol 2: 563-568, 1993.

22. Tahara E, Semba S and Tahara H: Molecular observations in gastric cancer. Semin Oncol 23: 307-315, 1996.

23. Mai M, Sakata Y, Kanamaru R, et al: A late phase II clinical study of RP56976 (docetaxel) in patients with advanced or recurrent gastric cancer: a cooperative study group trial (group B). Jpn J Chemother 26: 487-496, 1999.

24. Naitoh H, Kawaguchi A, Yamamoto H, et al: Measurement of docetaxel concentration in blood and ascites after drop infusion into each vessel and intraperitoneal cavity of gastric cancer. Jpn J Chemother 31: 2031-2034, 2004.

25. Hainsworth JD, Burris HA III, Erland JB, Thomas M and Greco F: A phase I trial of docetaxel administered by weekly infusion in patients with advanced refractory cancer. J Clin Oncol 16: 2164-2168, 1998.

26. Tabernero J, Climent MA, Lluch A, et al: A multicentre, randomised phase II study of weekly or 3-weekly docetaxel in patients with metastatic breast cancer. Ann Oncol 15: 1358-1365, 2004.

27. Loss WJ, Verweij J, Nooter K, Stoter G and Sparreboom A: Sensitive determination of docetaxel in human plasma by liquid-liquid extraction and reversed-phase high-performance liquid chromatography. J Chromatogr B Biomed 693: 437-441, 1997. 
28. Kuzuya T, Yamauchi M, Ito A, Hasegawa M, Hasegawa T and Nabeshima T: Pharmacokinetic characteristics of 5-fluorouracil and mitomycin $\mathrm{C}$ in intraperitoneal chemotherapy. J Pharm Pharmacol 46: 685-689, 1994.

29. Cancer JRSfG: The general rules for the gastric cancer study in surgery and pathology. I. Clinical classification. Jpn J Surg 11: 127-139, 1981.

30. Markman M, Rowinsky E, Hakes T, et al: Phase I trial of intraperitoneal taxol: a gynecologic oncology group study. J Clin Oncol 10: 1485-1491, 1992.

31. Taguchi T, Furue H, Niitani H, et al: Phase I clinical trial of RP56976 (Docetaxel) a new anticancer drug. Jpn J Cancer Chemother 21: 1997-2005, 1994.

32. DeGregorio MW, Lum BL and Holleran WM: Preliminary observations of intraperitoneal carboplatin pharmacokinetics during a phase I study of the Northern California Oncology Group. Cancer Chemother Pharmacol 18: 235-238, 1986.
33. Hiratsuka M, Furukawa H, Yasuda T, et al: Intraperitoneal Chemotherapy with Mitomycin C or Cisplatin. In: Multimodality Therapy for Gastric Cancer. Nakajima $T$ and Yamaguchi $T$ (eds). Springer, Tokyo, pp58-65, 1999.

34. Markman M: Intraperitoneal drug delivery of antineoplastics. Drugs 61: 1057-1065, 2001.

35. Francis P, Rowinsky E, Schneider J, et al: Phase I feasibility and pharmacologic study of weekly intraperitoneal paclitaxel: a gynecologic oncology group pilot study. J Clin Oncol 13: 2961-2967, 1995.

36. Morgan RJ, James HD, Synold T, et al: Phase I trial of intraperitoneal docetaxel in the treatment of advanced malignancies primary confined to the peritoneal cavity: dose-limiting toxicity and pharmacokinetics. Clin Cancer Res 9: 5896-5901, 2003.

37. Yokogawa K, Jin M, Furui N, et al: Disposition kinetics of taxanes after intraperitoneal administration in rats and influence of surfactant vehicles. J Pharm Pharmacol 56: 629$634,2004$. 